\title{
Geometrical Shaping of InGaN Light-Emitting Diodes by Laser Micromachining
}

\author{
W. Y. Fu, Student Member, IEEE, K. N. Hui, Student Member, IEEE, X. H. Wang, K. K. Y. Wong, Member, IEEE, \\ P. T. Lai, Senior Member, IEEE, and H. W. Choi, Senior Member, IEEE
}

\begin{abstract}
Geometrical shaping of InGaN light-emitting diodes (LEDs) by laser micromachining is introduced. The sapphire substrate is shaped with inclined sidewalls at $50^{\circ}$, serving as a prism favoring light redirection for out-coupling from the top window. Compared to conventional cuboid LEDs with a calculated light extraction efficiency $\eta_{\text {ext }}$ of $18.3 \%$, these shaped LEDs offers a pronounced increase in $\eta_{\text {ext }}$ of up to $85.2 \%$, verified by experimental results.
\end{abstract}

Index Terms-Laser micro-machining, light-emitting diodes (LEDs).

\section{INTRODUCTION}

$\mathbf{T}$ HE quantum-well structures within an InGaN light-emitting diode (LED) have now been optimized to offer internal quantum efficiencies exceeding 70\% [1], enabling widespread adaptation of LED products across a wide range of markets, including full-color panel displays, liquid-crystal display backlighting, and solid-state lighting amongst others [2]. Nevertheless, the potential of the LED emitter has yet to be fully unleashed due to limited light extraction efficiency. The large refractive index difference between nitride material $\left(n_{\mathrm{GaN}}=2.585\right)$ and air, giving rise to total internal reflection at the interfaces, is the major cause for the lower-than-expected external quantum efficiency. The refractive index difference $\Delta n$ of 0.8 between $\mathrm{GaN}$ and sapphire results in a further $\sim 24 \%$ of light being trapped within the sapphire substrate. Many solutions have been proposed and adopted to alleviate these issues, such as surface texturing [3], photonic crystals [4], [5], and geometrical chip-shaping [6]. The first two approaches deal with light that passes through the top surface of the chip. However, a significant portion of light is extracted from the sidewalls of the sapphire substrate; careful design of the overall chip geometry can effectively boost the light extraction efficiency, and modify the emission pattern. Conventional chip separation techniques such as dicing by diamond saw results in chips of cuboid geometry with a Lambertian emission pattern. Light extraction efficiency associated with this structure is often less than $20 \%$. Chips with parabolic or pyramidal geometry can potentially enhance light extraction. In a report by Krames et al. [7], AlInGaP-GaP LED chips of truncated-pyramidal

Manuscript received February 18, 2009; revised April 08, 2009. First published May 19, 2009; current version published July 15, 2009. This work was supported by a CERG grant of the Research Grant Council of Hong Kong (Project HKU 7121/06E).

The authors are with the Department of Electrical and Electronic Engineering, The University of Hong Kong, Hong Kong (e-mail: hwchoi@ hku.hk).

Color versions of one or more of the figures in this letter are available online at http://ieeexplore.ieee.org.

Digital Object Identifier 10.1109/LPT.2009.2022751 (a)

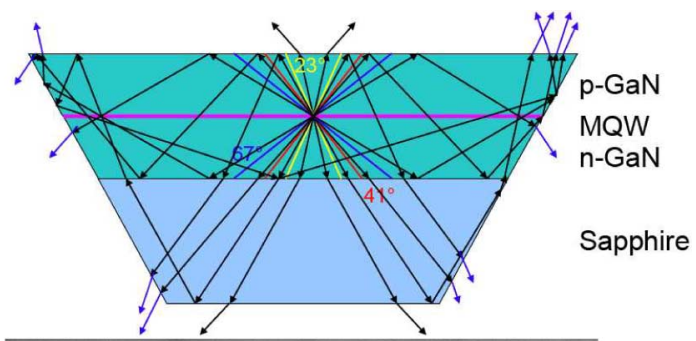

(b)

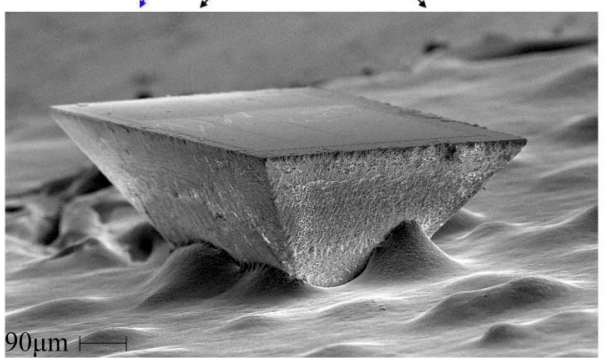

Fig. 1. (a) Schematic diagram showing exemplary light rays within a TP-LED Arrows in blue indicate the additional extracted rays due to inclined sidewalls. (b) SEM image showing an InGaN LED die of truncated pyramid geometry.

structure were realized using a beveled dicing technique, providing nearly twofold improvement in optical output. Similar improvements are expected from InGaN-sapphire LEDs, particularly due to the high transparency of the substrate. The inclined sidewall can serve as a reflector, redirecting light rays which would otherwise be trapped in a cuboid LED, for extraction through the top emitting area; examples of such rays are indicated in blue in Fig. 1(a).

In this letter, the dicing and shaping of InGaN LED chips in a single-step using laser micromachining is demonstrated. The chips are shaped into inverted pyramids (TPs). The performance of the chips is rigorously evaluated and compared with simulated results.

\section{EXPERIMENTAL DETAILS}

The conventional setup for laser micromachining comprises of an ultraviolet laser source, beam focusing optics and the sample placed on an $x-y$ motorized translation stage. The modified setup contains an additional beam-turning mirror between objective and sample, steering the beam away from the normal and striking the sample at oblique incidence. Details of this experimental setup are reported elsewhere [8]. The LEDs were fabricated using standard micro-fabrication procedures. The emissive regions of individual LED chips are $500 \mu \mathrm{m} \times 500 \mu \mathrm{m}$. Chips of truncated pyramidal geometry were diced and shaped by applying four successive oblique laser cuts onto the four sides of an LED. A scanning electron microscope (SEM) image of a TP-LED is shown in Fig. 1(b). 
The angular emission patterns of the devices were obtained by rotating a detector about its axis in the range of $-90^{\circ}$ to $90^{\circ}$, in steps of $2^{\circ}$, while the output powers were measured by placing the packaged chips within an integrating sphere, which is fiber-coupled to a calibrated optical spectrometer.

\section{RESULTS AND DISCUSSION}

A ray-tracing simulation coded in MATLAB was carried out to predict the emission patterns, taking into account light refraction based on Snell's law (in vector form)

$$
\begin{aligned}
\mathbf{r}_{r}= & \mathbf{r}_{\mathrm{in}}+\left[2\left(\mathbf{n} \cdot-\mathbf{r}_{r}\right)\right] \mathbf{n} \\
\mathbf{r}_{t}= & \left(n_{1} / n_{2}\right) \mathbf{r}_{\mathrm{in}}+\left\{\left(n_{1} / n_{2}\right)\left(\mathbf{n} \cdot-\mathbf{r}_{r}\right)\right. \\
& \left.+\sqrt{1-\left(n_{1} / n_{2}\right)^{2}\left[1-\left(\mathbf{n} \cdot-\mathbf{r}_{r}\right)^{2}\right]}\right\} \mathbf{n}
\end{aligned}
$$

where $\mathbf{r}_{\mathrm{in}}, \mathbf{r}_{r}$ and $\mathbf{r}_{t}$ are the normalized vectors of incident ray, reflected ray, and transmitted ray, respectively, $n_{1}$ and $n_{2}$ are the refractive indices, and $\mathbf{n}$ is the normalized normal vector of the interface; additionally, light reflectivity and transmissivity are evaluated based on Fresnel equation

$$
\begin{aligned}
& R_{s}=\left\{\frac{n_{1} \cos \left(\theta_{i}\right)-n_{2} \sqrt{1-\left[\left(n_{1} / n_{2}\right) \sin \left(\theta_{i}\right)\right]^{2}}}{n_{1} \cos \left(\theta_{i}\right)+n_{2} \sqrt{1-\left[\left(n_{1} / n_{2}\right) \sin \left(\theta_{i}\right)\right]^{2}}}\right\}^{2} \\
& R_{p}=\left\{\frac{n_{1} \sqrt{1-\left[\left(n_{1} / n_{2}\right) \sin \left(\theta_{i}\right)\right]^{2}}-n_{2} \cos \left(\theta_{i}\right)}{n_{1} \sqrt{1-\left[\left(n_{1} / n_{2}\right) \sin \left(\theta_{i}\right)\right]^{2}}+n_{2} \cos \left(\theta_{i}\right)}\right\}^{2}
\end{aligned}
$$

where $R_{s}$ and $R_{p}$ are reflection coefficients of s-polarized and p-polarized light ray, and $\theta_{i}$ is the incident angle, whereby $\cos \left(\theta_{i}\right)$ can be obtained by the term $\left(\mathbf{n} \cdot-\mathbf{r}_{r}\right)$ as used in (1) and (2). The absorption rule is

$$
I=I_{o} \exp (-\alpha L)
$$

where $I_{o}$ and $I$ are the intensities at the initial and final points of the light path, $\alpha$ the absorption coefficient $\left(\alpha_{\mathrm{GaN}}=150 \mathrm{~cm}^{-1}\right.$ was used in the simulation), and $L$ the distance travelled by each light ray.

The active region of an LED is modeled by a $50 \times 50$ array of point sources. The light extraction efficiency is evaluated by summing the intensities of light rays escaping from the LED divided by total intensities of light rays emitted

$$
\eta=\sum_{\theta, \phi} f\left(I_{\theta, \phi}\right) / \sum_{\theta, \phi} I_{\theta, \phi}
$$

where $I$ is the light intensity emitted at a specific angle $(\theta, \varphi)$, and $f(I)$ the light intensity extracted at that angle. A plot of light extraction efficiency as a function of inclination angle is plotted in Fig. 2. As observed from the graph, the light extraction efficiency for a cuboid structure $\left(90^{\circ}\right)$ is $18.3 \%$, rising to $33.9 \%$ for an inclination angle of $50^{\circ}$. Further increase in inclination angle results in little further improvement, while the cutting efficiency is reduced. Therefore, $50^{\circ}$ is deemed to be the optimal choice.

To obtain the radiation pattern, the number of light rays as a function of angle is plotted at a distance of $50 \mathrm{~mm}$ from the center of LED. The vector representing each corresponding light ray is thus

$$
\mathbf{r}_{\mathrm{ang}}=\mathbf{p}+u \mathbf{r}_{e}
$$

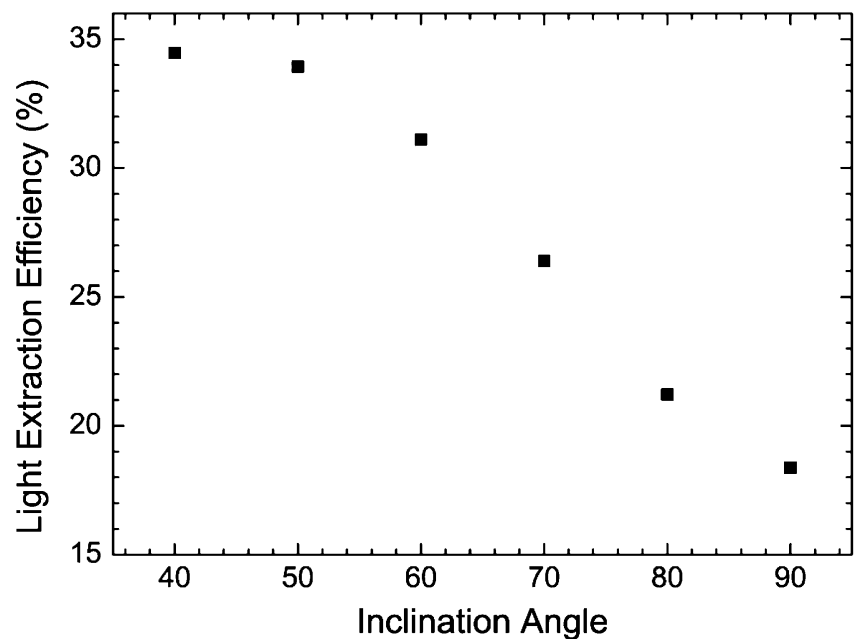

Fig. 2. Light extraction efficiency of inclined sidewall LED versus inclination angle of sidewall.

where $\mathbf{r}_{e}$ is the normalized vector of a ray extracted from the LED, and $\mathbf{p}$ is the position at which the light ray is extracted. $u$ can be evaluated by solving

$$
\left|\mathbf{p}+u \mathbf{r}_{e}\right|=d
$$

where $d$ is the distance between the LED center point and the point of detection, i.e., $50 \mathrm{~mm}$.

Based on the emission pattern obtained from the ray-tracing results, a larger fraction of light can be extracted from an LED with inclined sidewall compared to an LED of cuboid geometry. Photons which do not fall within the original escape cone [within the yellow boundary in Fig. 1(a)] can now find escape pathways through the inclined sidewall, or are redirected into the escape cone through multiple reflections on this inclined interface which acts as a mirror.

Experimental angular emission pattern of devices with different geometries are plotted in Fig. 3(a). These plots are normalized to the intensities at normal. The experimental data are compared with the theoretical calculations in Fig. 3(b) and (c). As observed in Fig. 3(b), the cuboid LED behaves as a Lambertian source as expected, with an emission half-angle of about $60^{\circ}$. The emission pattern of the TP-LED, as illustrated in Fig. 3(c), is widened, with half-angle extending to $65^{\circ}$. The enhancement of light emission occurs mainly at angles between $30^{\circ}$ and $60^{\circ}$ with respect to the normal, attributed to light redirection due to the inclined sidewall acting like a prism. Note that the inclined sidewall only reflects light rays incident beyond its critical angle.

In addition, simulation results indicate an overall increase in light extraction efficiency of TP-LEDs $85.2 \%$, compared to the light extraction efficiency of the reference cuboid device of $18.3 \%$, including the contributions of sidewall light emission. Operation images of a cuboid LED and a TP-LED are shown in Fig. 4(a) and (b), respectively, while their light output-current $(L-I)$ characteristics are plotted in Fig. 5. At lower driving currents $(<50 \mathrm{~mA})$, the average light enhancement factor is $88.6 \%$, consistent with our theoretical prediction of $85.2 \%$. Such significant improvement to light extraction efficiency highlights the effectiveness of geometrical chip-shaping, particularly with our approach based on laser micromachining. It is noted that the 


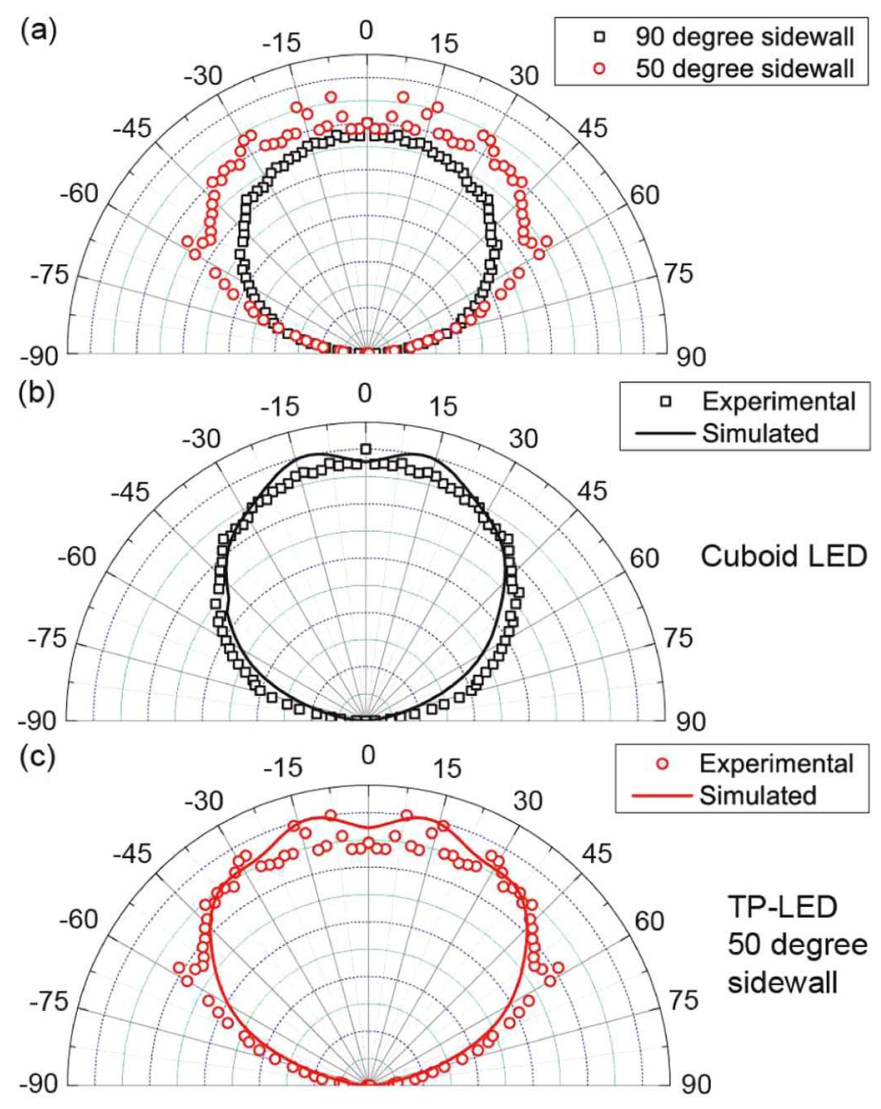

Fig. 3. (a) Shows the experimental angular emission patterns comparing LEDs of cuboid geometry (black line with square marker) and TP geometry (red line with circle marker). The experimental data are compared with simulated plots in (b) for cuboid LED, (c) for TP-LED.

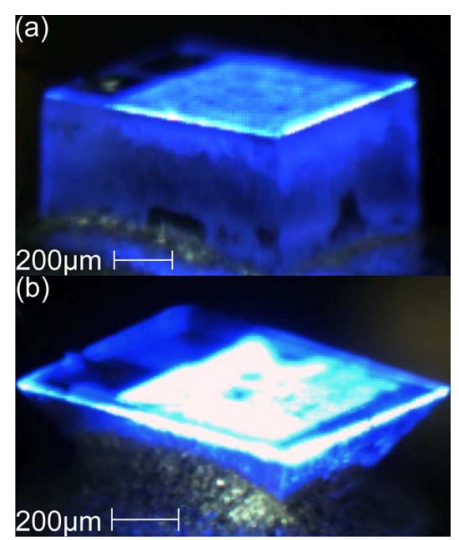

Fig. 4. Optical microphotographs showing (a) cuboid LED and (b) TP-LED biased at $10 \mathrm{~mA}$.

light output of the TP-LED begins saturating at $I>50 \mathrm{~mA}$, which is attributed to joule heating effect. Shaping chips into the TP geometry reduces the surface area of the base at the same time. Heat is dissipated from the chip to the package via this interface; a smaller contact area impedes heat transfer, thereby increasing the junction temperature, accounting for the nonlinearity at higher temperatures. This may be alleviated by ex-

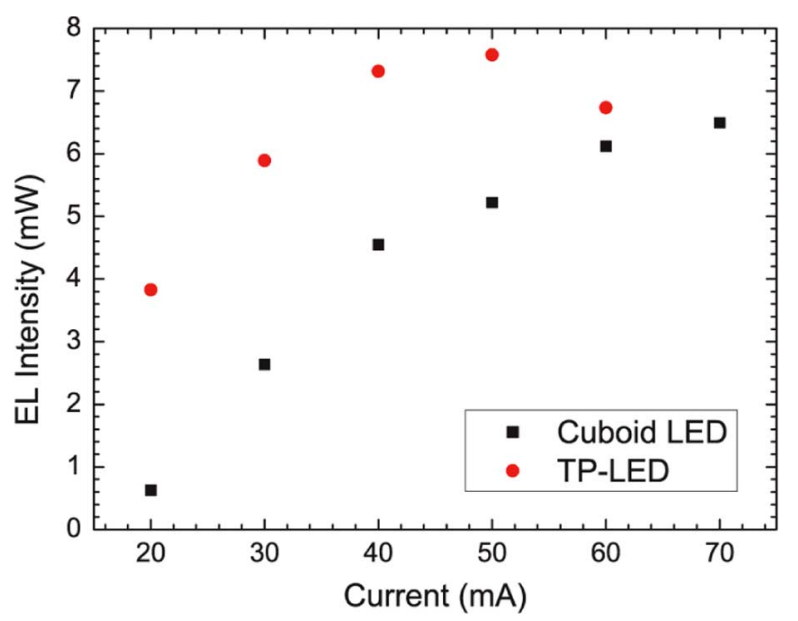

Fig. 5. $L-I$ curve comparing the performances of TP-LED and cuboid LED.

tending the coverage of the die-bonding epoxy to the inclined sidewalls, thereby increasing the effective die contact area.

\section{CONCLUSION}

In summary, LEDs of truncated pyramidal geometry shaped by laser micromachining were demonstrated. The inclined sidewalls served as a reflector to redirect laterally propagating photons into the escape cone. Devices of TP-geometry emit with a wider divergence angle. The calculated light extraction efficiency of a TP-LED increases by $85.2 \%$, compared to the absolute extraction efficiency of a cuboid LED of $18.3 \%$. Experimentally, an optical output enhancement of $88.6 \%$ was measured from the fabricated devices.

\section{REFERENCES}

[1] T. Akasaka, H. Gotoh, T. Saito, and T. Makimoto, "High luminescent efficiency of InGaN multiple quantum wells grown on InGaN underlying layers," Appl. Phys. Lett., vol. 85, pp. 3089-3091, Oct. 2004.

[2] E. F. Schubert and J. K. Kim, "Solid-state light sources getting smart," Science, vol. 308, pp. 1274-1278, May 2005.

[3] D. S. Han, J. Y. Kim, S. I. Na, S. H. Kim, K. D. Lee, B. Kim, and S. J. Park, "Improvement of light extraction efficiency of flip-chip lightemitting diode by texturing the bottom side-surface of sapphire substrate," IEEE Photon. Technol. Lett., vol. 18, no. 13, pp. 1406-1408, Jul. 1, 2006.

[4] S. H. Kim, K. D. Lee, J. Y. Kim, M. K. Kwon, and S. J. Park, "Fabrication of photonic crystal structures on light emitting diodes by nanoimprint lithography," Nanotechnology, vol. 18, p. 055306, Feb. 2007.

[5] W. N. Ng, C. H. Leung, P. T. Lai, and H. W. Choi, "Photonic crystal light-emitting diodes fabricated by microsphere lithography," Nanotechnology, vol. 19, p. 255302 , Jun. 2008.

[6] C. C. Kao, H. C. Kuo, H. W. Huang, J. T. Chu, Y. C. Peng, Y. L. Hsieh, C. Y. Luo, S. C. Wang, C. C. Yu, and C. F. Lin, "Light-output enhancement in a nitride-based light-emitting diode with $22^{\circ}$ undercut sidewalls," IEEE Photon. Technol. Lett., vol. 17, no. 1, pp. 19-21, Jan. 2005.

[7] M. R. Krames, M. Ochiai-Holcomb, G. E. Hofler, C. Carter-Coman, E. I. Chen, I. H. Tan, P. Grillot, N. F. Gardner, H. C. Chui, J. W. Huang, S. A. Stockman, F. A. Kish, M. G. Craford, T. S. Tan, C. P. Kocot, M. Hueschen, J. Posselt, B. Loh, G. Sasser, and D. Collins, "High-power truncated-inverted-pyramid (AlxGa1-x)(0.5)In0.5P/GaP light-emitting diodes exhibiting $>50 \%$ external quantum efficiency," Appl. Phys. Lett., vol. 75, pp. 2365-2367, Oct. 1999.

[8] X. H. Wang, P. T. Lai, and H. W. Choi, "Laser micromachining of optical microstructures with inclined sidewall profile," J. Vacuum Sci. Technol. B, vol. 27, pp. 1048-1052, May 2009. 